\title{
Draft Genome Sequence of the Agarase-Producing Sphingomonas sp. MCT13
}

\author{
Marco M. D'Andrea ${ }^{1,2 *}$, Nagaia Ciacci ${ }^{1,3}$, Vincenzo Di Pilato ${ }^{4}$, Gian M. Rossolini ${ }^{1,2,5}$ and \\ Maria C. Thaller ${ }^{3}$ \\ ${ }^{1}$ Department of Medical Biotechnologies, University of Siena, Siena, Italy, ${ }^{2}$ Department of Experimental and Clinical \\ Medicine, University of Florence, Florence, Italy, ${ }^{3}$ Department of Biology, University of Rome Tor Vergata, Rome, Italy, \\ ${ }^{4}$ Department of Surgery and Translational Medicine, University of Florence, Florence, Italy, ${ }^{5}$ Clinical Microbiology and Virology \\ Unit, Florence Careggi University Hospital, Florence, Italy
}

Keywords: Sphingomonas, agarase, Sphingomonadaceae, Sphingomonadales, draft genome, draft assemblies

\section{INTRODUCTION}

\section{OPEN ACCESS}

Edited by:

Jyoti Prakash Maity,

National Chung Cheng University,

Taiwan

Reviewed by:

Nitin Kumar Singh,

NASA Jet Propulsion

Laboratory-Caltech, USA

Sukalyan Chakraborty,

Birla Institute of Technology, Mesra,

India

*Correspondence:

Marco M. D'Andrea

marcomaria.dandrea@unifi.it

Specialty section:

This article was submitted to

Groundwater Resources and

Management

a section of the journal

Frontiers in Environmental Science

Received: 30 November 2016

Accepted: 27 February 2017

Published: 16 March 2017

Citation:

D'Andrea MM, Ciacci N, Di Pilato V, Rossolini GM and Thaller MC (2017) Draft Genome Sequence of the Agarase-Producing Sphingomonas sp. MCT13. Front. Environ. Sci. 5:9

doi: 10.3389/fenvs.2017.00009
The genus Sphingomonas, originally proposed by Yabuuchi et al., was subsequently amended and is now subdivided into four genera: Sphingomonas sensu stricto, Sphingobium, Novosphingobium, and Sphingopyxis (Yabuuchi et al., 1990; Takeuchi et al., 2001). Sphingomonads have gained particular attention for their unique abilities to degrade a variety of compounds, including pollutants produced by industrial processes, polycyclic aromatic hydrocarbons $(\mathrm{PAH})$ and dibenzofurans, and other toxic chemicals such as insecticides, herbicides, and heavy metals (Leys et al., 2004). Due to these features, sphingomonads are of great interest for bioremediation purposes, and have been previously exploited for the decontamination of groundwater systems from chemical pollutants of anthropogenic nature such as herbicides (Samuelsen et al., 2017), pentachlorophenol (Yang and Lee, 2008), and heavy metals (Vílchez et al., 2007). Agarose is a neutral linear polymer constituting the main fraction of agar produced by red macroalgae (Kim et al., 2016), which is widely used as a starting compound for the production of agar-derived oligosaccharides at industrial level (Yun et al., 2016). Enzymatic saccharification of agarose has a number of remarkable advantages over traditional acid degradation methods, such as the obtainment of well-defined chemical products, lower environmental pollution, and low energy costs (Fu and Kim, 2010).

Sphingomonas sp. MCT13 was isolated from a water sample collected in April 2002 at an artificial basin at the Experimental Ecology and Acquaculture Laboratories (Tor Vergata, Rome, Italy). The strain was able to form evident pitting on agar plates, suggesting the ability to degrade agar by the production of agarase activity, a feature that has not been previously reported for sphingomonads, and has till now been reported only twice in $\alpha$-Proteobacteria isolated from marine environments (Hosoda and Sakai, 2006; Kang and Lee, 2009). Here, we report on the draft genome sequence of the putative novel species Sphingomonas sp. MCT13, providing also the results of preliminary bioinformatic analyses that suggest gene candidates of potential interest in either bioremediation or for industrial applications in the field of complex carbohydrates degradation.

\section{MATERIALS AND METHODS}

\section{Isolation of Sphigomonas sp. MCT13}

A water sample $(1.2 \mathrm{~L})$ was collected from a drainage ditch within a disused system of constructed wetlands. The ditch flows through uncultivated land, within the Experimental Ecology and Aquaculture Laboratories area of the University of Rome Tor Vergata, at the south eastern outskirts of the town. Aliquots (10 $\mu \mathrm{L}$ each) of this water sample have been plated on Tryptic-Soy, ZoBell, 
and water agar plates and incubated at room temperature until the appearance of colonies. An isolated colony grown on the ZoBell plate was picked up, identified at the genus level by $16 \mathrm{~S}$ rDNA sequencing as Sphingomonas sp., and subjected to further analysis.

\section{DNA Extraction and Sequencing}

Sphigomonas sp. MCT13 was grown overnight at $35^{\circ} \mathrm{C}$ in TrypticSoy agar plates. A single colony was inoculated in LB broth and grown overnight at $35^{\circ} \mathrm{C}$. Bacterial DNA was extracted using the phenol-chloroform method (Sambrook and Russell, 2001) and then subjected to whole-genome sequencing with a MiSeq platform (Illumina Inc., San Diego, CA), using a $2 \times 250$ pairedend approach.

\section{Genome Assembly and Annotation}

De novo assembly was performed by using SPAdes 3.8 software (Bankevich et al., 2012) using default parameters. Scaffolds characterized by a length $\leq 200$ bp were removed. The quality of genome assembly was checked by read mapping performed with SAMtools (Li et al., 2009) and by BLASTn comparison with genomes of other members of the Sphingomonadaceae family to assess the collinearity of selected gene clusters. Automated annotation of the draft genome sequence has then been performed with NCBI Prokaryotic Genome Annotation Pipeline (PGAP) web-service available at NCBI (http://www.ncbi.nlm.nih.gov/genome/annotation_ prok).

\section{Bioinformatic Analysis}

Analysis of the $16 \mathrm{~S}$ rDNA sequences were performed as previously described by using a custom database of reference $16 \mathrm{~S}$ rDNA sequences (Leys et al., 2004). Phylogenetic relationships were assessed by using the ANIb method implemented in the JSpecies software V1.2.1 (Richter and Rossello-Mora, 2009). Detection of acquired antimicrobial resistance genes was carried out with ResFinder V2.1 (Zankari et al., 2012), while plasmid replicons were searched by using PlasmidFinder (Carattoli et al., 2014). The presence of prophages was investigated with the PHAST web-service (Zhou et al., 2011).

\section{RESULTS}

In total, 1,787,804 reads were obtained and assembled into 102 scaffolds ( $>200$ bp in size) having a total length of $4,108,924$ bp and characterized by a $\mathrm{N}_{50}$ of $147,599 \mathrm{bp}$ and an $\mathrm{L}_{50}$ of 11 . Genome raw coverage was $\approx 215 \mathrm{X}$. The average GC content was $65.3 \%$. A total of 3,851 CDS, 48 tRNAs, and 3 complete rRNAs were annotated by the PGAP web-service. Phylogenetic analysis performed as previously described (Leys et al., 2004) using a set of 40 reference 16S rDNA sequences from Sphingomonas sp., revealed that Sphingomonas sp. MCT13 shows the highest identity (98\%) with Sphingomonas sp. B101/7, representative of a putative new species.
Analysis of the whole genome of Sphingomonas sp. MCT13 in comparison with those of other Sphingomonas sp. deposited in the INSDC databases, by using the ANI (Average Nucleotide Identity) method, showed that Sphingomonas koreensis NBRC 16723 is the closest homolog, with an ANIb value of 78.3. This result supports the hypothesis that Sphingomonas sp. MCT13 belongs to a novel species, given the fact that cut-off values of ANIb for species delineation are $<95 \%$ (Goris et al., 2007).

ResFinder revealed the absence in Sphingomonas sp. MCT13 of any acquired resistance gene, while PlasmidFinder did not detect the presence of known plasmid replicon type. PHAST revealed the presence of two incomplete and one putative prophages. Detailed analysis of the PGAP annotation of the MCT13 draft genome revealed the presence of three hypothetical agarase-encoding genes (Accession numbers: ODP38961.1, ODP36587.1, and ODP36570.1), coding for enzymes displaying 60\% identity with a hypothetical agarase from Cellvibrio sp. BR, 48\% identity with a hypothetical beta-agarase from Pseudoalteromonas sp. BSi20429, and 53\% identity with an hypothetical agarase from Gilvimarinus agarilyticus (Lee et al., 2015), respectively. Interestingly, ODP38961.1 and ODP36570.1 displayed also 48 and 53\% protein identity, respectively, with a previously characterized exo-beta-agarase (Accession number 4BQ2_A) from the marine bacterium Saccharophagus degradans (Pluvinage et al., 2013), while ODP36587.1 displayed also a 31\% identity with a beta-porphyranase identified in the gut bacterium Bacteroides plebeius (Hehemann et al., 2012). Further work is currently ongoing to characterize these enzymes. Results of annotation from PGAP revealed also the presence in Sphingomonas sp. MCT13 of several gene clusters putatively associated to the degradation of $\mathrm{PAH}$. In particular the presence of genes encoding homologs of fldZAB/fldYXWVUT and flnA1A2 gene products of Sphingomonas sp. LB126 (Accession No. AJ277295.1 and EU024110.1, respectively; Wattiau et al., 2001; Schuler et al., 2008), suggests the ability of Sphingomonas sp. MCT13 to degrade fluorene.

The capability of Sphingomonas sp. MCT13 to degrade agar and possibly toxic $\mathrm{PAH}$, together with the lack of any known antibiotic resistance gene, make this strain potentially interesting for industrial applications in the field of complex carbohydrates degradation or for bioremediation purposes.

The complete genome sequence of Sphingomonas sp. MCT13 was deposited at DDBJ/EMBL/GenBank databases under the accession no. MDDS00000000. The version described in this paper is the version MDDS01000000. Read data were deposited in the NCBI SRA database under BioProject ID PRJNA338394 (experiment SRR5139223).

\section{AUTHOR CONTRIBUTIONS}

NC performed DNA extraction. DNA sequencing has been performed by VDP. Data analysis has been performed by MD, NC, and MT. MD, GR, and MT contributed to the writing and the editing of manuscript. 


\section{REFERENCES}

Bankevich, A., Nurk, S., Antipov, D., Gurevich, A. A., Dvorkin, M., Kulikov, A. S., et al. (2012). SPAdes: a new genome assembly algorithm and its applications to single-cell sequencing. J. Comput. Biol. 19, 455-477. doi: $10.1089 / \mathrm{cmb} .2012 .0021$

Carattoli, A., Zankari, E., García-Fernández, A., Voldby, L. M., Lund, O., Villa, L., et al. (2014). In silico detection and typing of plasmids using PlasmidFinder and plasmid multilocus sequence typing. Antimicrob. Agents Chemother. 58, 3895-3903. doi: 10.1128/AAC.02412-14

Fu, X. T., and Kim, S. M. (2010). Agarase: review of major sources, categories, purification method, enzyme characteristics and applications. Mar. Drugs 8, 200-218. doi: $10.3390 / \mathrm{md} 8010200$

Goris, J., Konstantinidis, K. T., Klappenbach, J. A., Coenye, T., Vandamme, P., and Tiedje, J. M. (2007). DNA-DNA hybridization values and their relationship to whole genome sequence similarities. Int. J. Syst. Evol. Microbiol. 57, 81-91. doi: 10.1099/ijs.0.64483-0

Hehemann, J. H., Kelly, A. G., Pudlo, N. A., Martens, E. C., and Boraston, A. B. (2012). Bacteria of the human gut microbiome catabolize red seaweed glycans with carbohydrate-active enzyme updates from extrinsic microbes. Proc. Natl. Acad. Sci. U.S.A. 109, 19786-19791. doi: 10.1073/pnas.1211002109

Hosoda, A., and Sakai, M. (2006). Isolation of Asticcacaulis sp. SA7, a novel agardegrading alphaproteobacterium. Biosci. Biotechnol. Biochem. 70, 722-725. doi: $10.1271 /$ bbb. 70.722

Kang, H. S., and Lee, S. D. (2009). Hirschia maritima sp. nov., isolated from seawater. Int. J. Syst. Evol. Microbiol. 59, 2264-2268. doi: 10.1099/ijs.0.008326-0

Kim, J. H., Yun, E. J., Seo, N., Yu, S., Kim, D. H., Cho, K. M., et al. (2016). Enzymatic liquefaction of agarose above the sol-gel transition temperature using a thermostable endo-type $\beta$-agarase, Aga16B. Appl. Microbiol. Biotechnol. 101, 1111-1120. doi: 10.1007/s00253-016-7831-y

Lee, Y., Lee, S. J., Park, G. H., Heo, S. J., Umasuthan, N., Kang, D. H., et al. (2015). Draft genome of agar-degrading marine bacterium Gilvimarinus agarilyticus JEA5. Mar. Genomics 21, 13-14. doi: 10.1016/j.margen.2015.03.001

Leys, N. M., Ryngaert, A., Bastiaens, L., Verstraete, W., Top, E. M., and Springael, D. (2004). Occurrence and phylogenetic diversity of Sphingomonas strains in soils contaminated with polycyclic aromatic hydrocarbons. Appl. Environ. Microbiol. 70, 1944-1955. doi: 10.1128/AEM.70.4.1944-1955.2004

Li, H., Handsaker, B., Wysoker, A., Fennell, T., Ruan, J., Homer, N., et al. (2009). The sequence alignment/map format and SAMtools. Bioinformatics 25, 2078-2079. doi: 10.1093/bioinformatics/btp352

Pluvinage, B., Hehemann, J. H., and Boraston, A. B. (2013). Substrate recognition and hydrolysis by a family 50 exo-beta-agarase, Aga50D, from the marine bacterium Saccharophagus degradans. J. Biol. Chem. 288, 28078-28088. doi: 10.1074/jbc.M113.491068

Richter, M., and Rosselló-Móra, R. (2009). Shifting the genomic gold standard for the prokaryotic species definition. Proc. Natl. Acad. Sci. U.S.A. 106, 19126-19131. doi: 10.1073/pnas.0906412106

Sambrook, J., and Russell, D. W. (2001). Molecular Cloning: A Laboratory Manual, $3 r d E d n$. Cold Spring Harbor, NY: Cold Spring Harbor Laboratory Press.

Samuelsen, E. D., Badawi, N., Nybroe, O., Sørensen, S. R., and Aamand, J. (2017). Adhesion to sand and ability to mineralise low pesticide concentrations are required for efficient bioaugmentation of flow-through sand filters. Appl. Microbiol. Biotechnol. 101, 411-421. doi: 10.1007/s00253-016-7909-6

Schuler, L., Ní Chadhain, S. M., Jouanneau, Y., Meyer, C., and Zylstra, G. J., Hols, P., et al. (2008). Characterization of a novel angular dioxygenase from fluorene-degrading Sphingomonas sp. strain LB126. Appl. Environ. Microbiol. 74, 1050-1057. doi: 10.1128/AEM.01627-07

Takeuchi, M., Hamana, K., and Hiraishi, A. (2001). Proposal of the genus Sphingomonas sensu stricto and three new genera, Sphingobium, Novosphingobium and Sphingopyxis, on the basis of phylogenetic and chemotaxonomic analyses. Int. J. Syst. Evol. Microbiol. 51, 1405-1417. doi: 10.1099/00207713-51-4-1405

Vílchez, R., Pozo, C., Gómez, M. A., Rodelas, B., and González-López, J. (2007). Dominance of sphingomonads in a copperexposed biofilm community for groundwater treatment. Microbiology (Reading, Engl). 153, 325-337. doi: 10.1099/mic.0.2006/002139-0

Wattiau, P., Bastiaens, L., van Herwijnen, R., Daal, L., Parsons, J. R., Renard, M. E., et al. (2001). Fluorene degradation by Sphingomonas sp. LB126 proceeds through protocatechuic acid: a genetic analysis. Res. Microbiol. 152, 861-872. doi: 10.1016/S0923-2508(01)01269-4

Yabuuchi, E., Yano, I., Oyaizu, H., Hashimoto, Y., Ezaki, T., and Yamamoto, H. (1990). Proposals of Sphingomonas paucimobilis gen. nov. and comb. nov., Sphingomonas parapaucimobilis sp. nov., Sphingomonas yanoikuyae sp. nov., Sphingomonas adhaesiva sp. nov., Sphingomonas capsulata comb. nov., and two genospecies of the genus Sphingomonas. Microbiol. Immunol. 34, 99-119. doi: 10.1111/j.1348-0421.1990.tb00996.x

Yang, C. F., and Lee, C. M. (2008). Pentachlorophenol contaminated groundwater bioremediation using immobilized Sphingomonas cells inoculation in the bioreactor system. J. Hazard. Mater. 152, 159-165. doi: 10.1016/j.jhazmat.2007.06.102

Yun, E. J., Kim, H. T., Cho, K. M., Yu, S., Kim, S., Choi, I. G., et al. (2016). Pretreatment and saccharification of red macroalgae to produce fermentable sugars. Bioresour. Technol. 199, 311-318. doi: 10.1016/j.biortech.2015. 08.001

Zankari, E., Hasman, H., Cosentino, S., Vestergaard, M., Rasmussen, S., Lund, O., et al. (2012). Identification of acquired antimicrobial resistance genes. J. Antimicrob. Chemother. 67, 2640-2644. doi: 10.1093/jac/dks261

Zhou, Y., Liang, Y., Lynch, K. H., Dennis, J. J., and Wishart, D. S. (2011). PHAST: a fast phage search tool. Nucleic Acids Res. 39(Web Server issue), W347-W352. doi: $10.1093 / \mathrm{nar} / \mathrm{gkr} 485$

Conflict of Interest Statement: The authors declare that the research was conducted in the absence of any commercial or financial relationships that could be construed as a potential conflict of interest.

Copyright (๑ 2017 D'Andrea, Ciacci, Di Pilato, Rossolini and Thaller. This is an open-access article distributed under the terms of the Creative Commons Attribution License (CC BY). The use, distribution or reproduction in other forums is permitted, provided the original author(s) or licensor are credited and that the original publication in this journal is cited, in accordance with accepted academic practice. No use, distribution or reproduction is permitted which does not comply with these terms. 